\title{
SOME CODING THEOREMS ON GENERALIZED HAVRDA-CHARVAT AND TSALLIS'S ENTROPY
}

\author{
SATISH KUMAR AND ARUN CHOUDHARY
}

\begin{abstract}
A new measure $L_{\alpha}^{\beta}$, called average code word length of order $\alpha$ and type $\beta$ has been defined and its relationship with a result of generalized Havrda-Charvat and Tsallis's entropy has been discussed. Using $L_{\alpha}^{\beta}$, some coding theorem for discrete noiseless channel has been proved.
\end{abstract}

\section{Introduction}

Throughout the paper $\mathbb{N}$ denotes the set of the natural numbers and for $N \in \mathbb{N}$ we set

$$
\Delta_{N}=\left\{\left(p_{1}, \ldots, p_{N}\right) / p_{i} \geq 0, i=1, \ldots, N, \sum_{i=1}^{N} p_{i}=1\right\} .
$$

In case there is no rise to misunderstanding we write $P \in \Delta_{N}$ instead of $\left(p_{1}, \ldots, p_{N}\right) \in \Delta_{N}$.

In case $N \in \mathbb{N}$ the well-known Shannon entropy is defined by

$$
H(P)=H\left(p_{1}, \ldots, p_{N}\right)=-\sum_{i=1}^{N} p_{i} \log \left(p_{i}\right) \quad\left(\left(p_{1}, \ldots, p_{N}\right) \in \Delta_{N}\right),
$$

where the convention $0 \log (0)=0$ is adapted, (see Shannon [19]).

Throughout this paper, $\sum$ will stand for $\sum_{i=1}^{N}$ unless otherwise stated and logarithms are taken to the base $D(D>1)$.

Let a finite set of $\mathrm{N}$ input symbols

$$
X=\left\{x_{1}, x_{2}, \ldots, x_{N}\right\}
$$

be encoded using alphabet of $D$ symbols, then it has been shown by Feinstien [6] that there is a uniquely decipherable code with lengths $n_{1}, n_{2}, \ldots, n_{N}$ if and only if the Kraft inequality holds that is,

$$
\sum_{i=1}^{N} D^{-n_{i}} \leq 1
$$

Corresponding author: Arun Choudhary.

2010 Mathematics Subject Classification. 94A15, 94A17, 94A24, $26 \mathrm{D} 15$.

Key words and phrases. Tsallis's entropy, codeword length, Kraft inequality, optimal code length and power probabilities. 
Where $D$ is the size of code alphabet.

Furthermore, if

$$
L=\sum_{i=1}^{N} n_{i} p_{i}
$$

is the average codeword length, then for a code satisfying (1.2), the inequality

$$
L \geq H(P)
$$

is also fulfilled and equality holds if and only if

$$
n_{i}=-\log _{D}\left(p_{i}\right)(i=1, \ldots, N)
$$

and that by suitable encoded into words of long sequences, the average length can be made arbitrarily close to $H(P)$, (see Feinstein [6]). This is Shannon's noiseless coding theorem.

By considering Renyi's entropy (see e.g. [18]), a coding theorem and analogous to the above noiseless coding theorem has been established by Campbell [3] and the authors obtained bounds for it in terms of $\mathrm{H}_{\alpha}(P)=\frac{1}{1-\alpha} \log _{D} \sum P_{i}^{\alpha}, \alpha>0(\neq 1)$. Kieffer [13] defined a class rules and showed $H_{\alpha}(P)$ is the best decision rule for deciding which of the two sources can be coded with expected cost of sequences of length $n$ when $n \rightarrow \infty$, where the cost of encoding a sequence is assumed to be a function of length only. Further, in Jelinek [10] it is shown that coding with respect to Campbell's mean length is useful in minimizing the problem of buffer overflow which occurs when the source symbol is produced at a fixed rate and the code words are stored temporarily in a finite buffer. Concerning Campbell's mean length the reader can consult [3].

Hooda and Bhaker considered in [9] the following generalization of Campbell's mean length:

$$
L^{\beta}(t)=\frac{1}{t} \log _{D}\left\{\frac{\sum p_{i}^{\beta} D^{-t n_{i}}}{\sum p_{i}^{\beta}}\right\}, \beta \geq 1
$$

and proved

$$
H_{\alpha}^{\beta}(P) \leq L^{\beta}(t)<H_{\alpha}^{\beta}(P)+1, \quad \alpha>0, \alpha \neq 1, \beta \geq 1
$$

under the condition

$$
\sum p_{i}^{\beta-1} D^{-n_{i}} \leq \sum p_{i}^{\beta}
$$

where $H_{\alpha}^{\beta}(P)$ is generalized entropy of order $\alpha=\frac{1}{1+t}$ and type $\beta$ studied by Aczel and Daroczy [1] and Kapur [11]. It may be seen that the mean codeword length (1.3) had been generalized parametrically and their bounds had been studied in terms of generalized measures of entropies. Here we give another generalization of (1.3) and study its bounds in terms of generalized entropy of order $\alpha$ and type $\beta$. 
Generalized coding theorems by considering different information measure under the condition of unique decipherability were investigated by several authors, see for instance the papers $[7,9,12,14,17,20]$.

In this paper we study some coding theorems by considering a new information measure depending on two parameters. Our motivation is -among others- that this quantity generalizes some information measures already existing in the literature such as the Tsallis (or Havrda-Charvat) entropy, (see [8] and [21]).

\section{Coding theorem}

Definition. Let $N \in \mathbb{N}$ be arbitrarily fixed, $\alpha, \beta>0, \alpha \neq 1$ be given real numbers. Then the information measure $H_{\alpha}^{\beta}: \Delta_{N} \rightarrow \mathbb{R}$ is defined by

$$
H_{\alpha}^{\beta}\left(p_{1}, \ldots, p_{N}\right)=\frac{1}{\alpha-1}\left(1-\frac{\sum_{i=1}^{N} p_{i}^{\alpha \beta}}{\sum_{j=1}^{N} p_{j}^{\beta}}\right)\left(\left(p_{1}, \ldots, p_{N}\right) \in \Delta_{N}\right) .
$$

Remark (i) When $\beta=1$, then the information measure $H_{\alpha}^{\beta}$ reduces to entropy,

$$
\text { i.e., } \quad H_{\alpha}\left(p_{1}, \ldots, p_{N}\right)=\frac{1}{\alpha-1}\left[1-\sum_{i=1}^{N} p_{i}^{\alpha}\right] \quad\left(\left(p_{1}, \ldots, p_{N}\right) \in \Delta_{N}\right) \text {. }
$$

The measure (2.2) was characterized by Havrda-Charvat [8], Vajda [22], Tsallis [21] and Daroczy [4] by different approaches.

(ii) When $\beta=1$ and $\alpha \rightarrow 1$, then the information measure $H_{\alpha}^{\beta}$ reduces to Shannon entropy,

$$
\text { i.e., } H(P)=-\sum p_{i} \log p_{i} \text {. }
$$

(iii) When $\alpha \rightarrow 1$, the information measure $H_{\alpha}^{\beta}$ is the entropy of the $\beta$-power distribution,

$$
\text { i.e., } \quad H^{\beta}(P)=-\frac{\sum_{i=1}^{N} p_{i}^{\beta} \log \left(p_{i}^{\beta}\right)}{\sum_{j=1}^{N} p_{j}^{\beta}}
$$

that was considered e.g. in Mathur-Mitter [16].

Definition. Let $N \in \mathbb{N}, \alpha, \beta>0, \alpha \neq 1$ be arbitrarily fixed, then the mean length $L_{\alpha}^{\beta}$ corresponding to the generalized information measure $H_{\alpha}^{\beta}$ is given by the formula

$$
L_{\alpha}^{\beta}=\frac{1}{\alpha-1}\left(1-\left(\frac{\sum_{i=1}^{N} p_{i}^{\beta} D^{-n_{i}\left(\frac{\alpha-1}{\alpha}\right)}}{\sum_{j=1}^{N} p_{j}^{\beta}}\right)^{\alpha}\right),
$$


where $\left(p_{1}, \ldots, p_{N}\right) \in \Delta_{N}$ and $D, n_{1}, n_{2}, \ldots, n_{N}$ are positive integers so that

$$
\sum_{i=1}^{N} D^{-n_{i}} \leq \sum_{j=1}^{N} p_{j}^{\beta}
$$

Remark (i) When $\beta=1$, (2.5) reduces to a mean codeword length,

$$
\text { i.e., } \quad L_{\alpha}=\frac{1}{\alpha-1}\left[1-\left\{\sum p_{i} D^{-n_{i}\left(\frac{\alpha-1}{\alpha}\right)}\right\}^{\alpha}\right] \text {. }
$$

(ii) When $\beta=1, \alpha \rightarrow 1$, (2.5) reduces to a mean code length $L=\sum_{i=1}^{N} n_{i} p_{i}$, defined in Shannon [19].

Also, we have used the condition (2.6) to find the bounds. It may be seen that the case $\beta=1$ inequality (2.6) reduces to (1.2).

We establish a result, that in a sense, provides a characterization of $H_{\alpha}^{\beta}(P)$ under the condition of (2.6).

Theorem 2.1. Let $\alpha, \beta>0, \alpha \neq 1$ be arbitrarily fixed real numbers, then for all integers $D>1$ inequality

$$
L_{\alpha}^{\beta} \geq H_{\alpha}^{\beta}(P)
$$

is fulfilled. Furthermore, equality holds if and only if

$$
n_{i}=-\log _{D}\left(\frac{p_{i}^{\alpha \beta}}{\frac{\sum_{i=1}^{N} p_{i}^{\alpha \beta}}{\sum_{j=1}^{N} p_{j}^{\beta}}}\right) .
$$

Proof. By reverse Hölder inequality, that is, if $N \in \mathbb{N}, \gamma>1$ and $x_{1}, \ldots, x_{N}, y_{1}, \ldots, y_{N}$ are positive real numbers then

$$
\left(\sum_{i=1}^{N} x_{i}^{\frac{1}{\gamma}}\right)^{\gamma} \cdot\left(\sum_{i=1}^{N} y_{i}^{-\frac{1}{(\gamma-1)}}\right)^{-(\gamma-1)} \leq \sum_{i=1}^{N} x_{i} y_{i}
$$

Let $\gamma=\frac{\alpha}{\alpha-1}, \quad x_{i}=\left(\frac{p_{i}^{\beta}}{\sum_{j=1}^{N} p_{j}^{\beta}}\right)^{\left(\frac{\alpha}{\alpha-1}\right)} D^{-n_{i}}, \quad y_{i}=\left(\frac{p_{i}^{\alpha \beta}}{\sum_{j=1}^{N} p_{j}^{\beta}}\right)^{\frac{1}{1-\alpha}}(i=1, \ldots, N)$.

Putting these values into (2.10), we get

$$
\left(\frac{\sum p_{i}^{\beta} D^{-n_{i}\left(\frac{\alpha-1}{\alpha}\right)}}{\sum_{j=1}^{N} p_{j}^{\beta}}\right)^{\frac{\alpha}{\alpha-1}}\left(\frac{\sum_{i=1}^{N} p_{i}^{\alpha \beta}}{\sum_{j=1}^{N} p_{j}^{\beta}}\right)^{\frac{1}{1-\alpha}} \leq \frac{\sum_{i=1}^{N} D^{-n_{i}}}{\sum_{j=1}^{N} p_{j}^{\beta}} \leq 1,
$$

where we used (2.6), too. This implies however that

$$
\left(\frac{\sum_{i=1}^{N} p_{i}^{\alpha \beta}}{\sum_{j=1}^{N} p_{j}^{\beta}}\right)^{\frac{1}{1-\alpha}} \leq\left(\frac{\sum_{i=1}^{N} p_{i}^{\beta} D^{-n_{i}\left(\frac{\alpha-1}{\alpha}\right)}}{\sum_{j=1}^{N} p_{j}^{\beta}}\right)^{\frac{\alpha}{1-\alpha}} .
$$


Here two cases arise

Case 1. When $0<\alpha<1$, then raising power $(1-\alpha)$ to both sides of (2.11), we have

$$
\left(\frac{\sum_{i=1}^{N} p_{i}^{\alpha \beta}}{\sum_{j=1}^{N} p_{j}^{\beta}}\right) \leq\left(\frac{\sum p_{i}^{\beta} D^{-n_{i}\left(\frac{\alpha-1}{\alpha}\right)}}{\sum_{j=1}^{N} p_{j}^{\beta}}\right)^{\alpha}
$$

we obtain the result (2.8) after simplification for $\frac{1}{\alpha-1}<0$ as $0<\alpha<1$. i.e.,

$$
L_{\alpha}^{\beta} \geq H_{\alpha}^{\beta}(P) \text {, when } 0<\alpha<1 \text {. }
$$

Case 2. When $\alpha>1$, then raising power $(1-\alpha)$ to both sides of $(2.11)$, we have

$$
\left(\frac{\sum_{i=1}^{N} p_{i}^{\alpha \beta}}{\sum_{j=1}^{N} p_{j}^{\beta}}\right) \geq\left(\frac{\sum p_{i}^{\beta} D^{-n_{i}\left(\frac{\alpha-1}{\alpha}\right)}}{\sum_{j=1}^{N} p_{j}^{\beta}}\right)^{\alpha}
$$

we obtain the result (2.8) after simplification for $\frac{1}{\alpha-1}>0$ as $\alpha>1$. i.e., $L_{\alpha}^{\beta} \geq H_{\alpha}^{\beta}(P)$, when $\alpha>1$.

Case 3. From (2.9) and after simplification, we get

$$
D^{-n_{i}\left(\frac{\alpha-1}{\alpha}\right)}=p_{i}^{\beta(\alpha-1)}\left(\frac{\sum_{i=1}^{N} p_{i}^{\alpha \beta}}{\sum_{j=1}^{N} p_{j}^{\beta}}\right)^{\left(\frac{1-\alpha}{\alpha}\right)} .
$$

This implies

$$
\left(\frac{\sum p_{i}^{\beta} D^{-n_{i}\left(\frac{\alpha-1}{\alpha}\right)}}{\sum_{j=1}^{N} p_{j}^{\beta}}\right)^{\alpha}=\left(\frac{\sum_{i=1}^{N} p_{i}^{\alpha \beta}}{\sum_{j=1}^{N} p_{j}^{\beta}}\right),
$$

which gives $L_{\alpha}^{\beta}=H_{\alpha}^{\beta}(P)$.

Theorem 2.2. Let $N \in \mathbb{N}, \alpha, \beta>0, \alpha \neq 1$ be fixed. Then there exist code length $n_{1}, \ldots, n_{N}$ so that

$$
H_{\alpha}^{\beta}(P) \leq L_{\alpha}^{\beta}<D^{1-\alpha} \mathrm{H}_{\alpha}^{\beta}(\mathrm{P})+\frac{1}{\alpha-1}\left(1-\mathrm{D}^{1-\alpha}\right)
$$

holds. Where $H_{\alpha}^{\beta}(P)$ and $L_{\alpha}^{\beta}$ are given by (2.1) and (2.5) respectively.

Proof. Due to the previous theorem,

$$
L_{\alpha}^{\beta}=H_{\alpha}^{\beta}(P)
$$

holds if and only if

$$
D^{-n_{i}}=\frac{p_{i}^{\alpha \beta}}{\frac{\sum_{i=1}^{N} p_{i}^{\alpha \beta}}{\sum_{j=1}^{N} p_{j}^{\beta}}}, \alpha>0, \alpha \neq 1, \beta>0,
$$


i.e., $n_{i}=-\log _{D} p_{i}^{\alpha \beta}+\log _{D}\left[\frac{\sum_{i=1}^{N} p_{i}^{\alpha \beta}}{\sum_{j=1}^{N} p_{j}^{\beta}}\right]$.

We choose the codeword lengths $n_{i}, i=1, \ldots, N$ in such a way that

$$
-\log _{D} p_{i}^{\alpha \beta}+\log _{D}\left[\frac{\sum_{i=1}^{N} p_{i}^{\alpha \beta}}{\sum_{j=1}^{N} p_{j}^{\beta}}\right] \leq n_{i}<-\log _{D} p_{i}^{\alpha \beta}+\log _{D}\left[\frac{\sum_{i=1}^{N} p_{i}^{\alpha \beta}}{\sum_{j=1}^{N} p_{j}^{\beta}}\right]+1
$$

is fulfilled for all $i=1, \ldots, N$.

From the left inequality of (2.14), we have

$$
D^{-n_{i}} \leq \frac{p_{i}^{\alpha \beta}}{\frac{\sum_{i=1}^{N} p_{i}^{\alpha \beta}}{\sum_{j=1}^{N} p_{j}^{\beta}}},
$$

taking sum over $i$, we get the generalized inequality (2.6). So there exists a generalized code with code lengths $n_{i}, i=1, \ldots, N$.

Case 1. Let $0<\alpha<1$, then (2.14) can be written as

$$
p_{i}^{\beta(\alpha-1)}\left(\frac{\sum_{i=1}^{N} p_{i}^{\alpha \beta}}{\sum_{j=1}^{N} p_{j}^{\beta}}\right)^{\frac{1-\alpha}{\alpha}} \leq \mathrm{D}^{-\mathrm{n}_{\mathrm{i}}\left(\frac{\alpha-1}{\alpha}\right)}<p_{i}^{\beta(\alpha-1)}\left(\frac{\sum_{i=1}^{N} p_{i}^{\alpha \beta}}{\sum_{j=1}^{N} p_{j}^{\beta}}\right)^{\frac{1-\alpha}{\alpha}} \mathrm{D}^{\frac{1-\alpha}{\alpha}} .
$$

Multiplying (2.16) throughout by $\frac{p_{i}^{\beta}}{\sum_{j=1}^{N} p_{j}^{\beta}}$ and then summing up from $i=1$ to $i=N$, we obtain inequality (2.13) after simplification with $\frac{\alpha}{\alpha-1}$,

$$
\text { i.e., } \begin{aligned}
\frac{1}{\alpha-1}\left(1-\left(\frac{\sum_{i=1}^{N} p_{i}^{\alpha \beta}}{\sum_{j=1}^{N} p_{j}^{\beta}}\right)\right) & \leq \frac{1}{\alpha-1}\left(1-\frac{\sum_{i=1}^{N} p_{i}^{\beta} D^{-n_{i}\left(\frac{\alpha-1}{\alpha}\right)}}{\sum_{j=1}^{N} p_{j}^{\beta}}\right)^{\alpha} \\
& <D^{1-\alpha}\left\{\frac{1}{\alpha-1}\left(1-\frac{\sum_{i=1}^{N} p_{i}^{\alpha \beta}}{\sum_{j=1}^{N} p_{j}^{\beta}}\right)\right\}+\frac{1}{\alpha-1}\left(1-D^{1-\alpha}\right)
\end{aligned}
$$

$H_{\alpha}^{\beta}(P) \leq L_{\alpha}^{\beta}<D^{1-\alpha} \mathrm{H}_{\alpha}^{\beta}(\mathrm{P})+\frac{1}{\alpha-1}\left(1-\mathrm{D}^{1-\alpha}\right)$, which gives (2.13).

Case 2. Let $\alpha>1$, then (2.14) can be written as

$$
p_{i}^{\beta(\alpha-1)}\left(\frac{\sum_{i=1}^{N} p_{i}^{\alpha \beta}}{\sum_{j=1}^{N} p_{j}^{\beta}}\right)^{\frac{1-\alpha}{\alpha}} \geq \mathrm{D}^{-\mathrm{n}_{\mathrm{i}}\left(\frac{\alpha-1}{\alpha}\right)}>p_{i}^{\beta(\alpha-1)}\left(\frac{\sum_{i=1}^{N} p_{i}^{\alpha \beta}}{\sum_{j=1}^{N} p_{j}^{\beta}}\right)^{\frac{1-\alpha}{\alpha}} \mathrm{D}^{\frac{1-\alpha}{\alpha}} .
$$

Multiplying (2.16) throughout by $\frac{p_{i}^{\beta}}{\sum_{j=1}^{N} p_{j}^{\beta}}$ and then summing up from $i=1$ to $i=N$, we obtain inequality (2.13) after simplification with $\frac{\alpha}{\alpha-1}$,

$$
\text { i.e., } \frac{1}{\alpha-1}\left(1-\left(\frac{\sum_{i=1}^{N} p_{i}^{\alpha \beta}}{\sum_{j=1}^{N} p_{j}^{\beta}}\right)\right) \leq \frac{1}{\alpha-1}\left(1-\frac{\sum_{i=1}^{N} p_{i}^{\beta} D^{-n_{i}\left(\frac{\alpha-1}{\alpha}\right)}}{\sum_{j=1}^{N} p_{j}^{\beta}}\right)^{\alpha}
$$




$$
<D^{1-\alpha}\left\{\frac{1}{\alpha-1}\left(1-\frac{\sum_{i=1}^{N} p_{i}^{\alpha \beta}}{\sum_{j=1}^{N} p_{j}^{\beta}}\right)\right\}+\frac{1}{\alpha-1}\left(1-D^{1-\alpha}\right)
$$

$H_{\alpha}^{\beta}(P) \leq L_{\alpha}^{\beta}<D^{1-\alpha} \mathrm{H}_{\alpha}^{\beta}(\mathrm{P})+\frac{1}{\alpha-1}\left(1-\mathrm{D}^{1-\alpha}\right)$, which gives (2.13).

Theorem 2.3. For arbitrary $N \in \mathbb{N}, \alpha, \beta>0, \alpha \neq 1$ and for every code word lengths $n_{i}, i=1, \ldots, N$ of Theorem 2.1, $L_{\alpha}^{\beta}$ can be made to satisfy,

$$
L_{\alpha}^{\beta} \geq H_{\alpha}^{\beta}(P)>H_{\alpha}^{\beta}(P) D+\frac{1}{\alpha-1}(1-D) .
$$

Proof. Suppose

$$
\bar{n}_{i}=-\log _{D}\left(\frac{p_{i}^{\alpha \beta}}{\frac{\sum_{i=1}^{N} p_{i}^{\alpha \beta}}{\sum_{j=1}^{N} p_{j}^{\beta}}}\right), \quad \alpha>0, \alpha \neq 1, \beta>0 .
$$

Clearly $\bar{n}_{i}$ and $\bar{n}_{i}+1$ satisfy 'equality' in Holder's inequality (2.10). Moreover, $\bar{n}_{i}$ satisfies (2.6). Suppose $n_{i}$ is the unique integer between $\bar{n}_{i}$ and $\bar{n}_{i}+1$, then obviously, $n_{i}$ satisfies (2.6).

Since $\alpha>0(\neq 1)$, we have

$$
\left(\frac{\sum_{i=1}^{N} p_{i}^{\beta} D^{-n_{i}\left(\frac{\alpha-1}{\alpha}\right)}}{\sum_{j=1}^{N} p_{j}^{\beta}}\right)^{\alpha} \leq\left(\frac{\sum_{i=1}^{N} p_{i}^{\beta} D^{-\bar{n}_{i}\left(\frac{\alpha-1}{\alpha}\right)}}{\sum_{j=1}^{N} p_{j}^{\beta}}\right)^{\alpha}<D\left(\frac{\sum_{i=1}^{N} p_{i}^{\beta} D^{-\bar{n}_{i}\left(\frac{\alpha-1}{\alpha}\right)}}{\sum_{j=1}^{N} p_{j}^{\beta}}\right)^{\alpha} .
$$

Since $\left(\frac{\sum_{i=1}^{N} p_{i}^{\beta} D^{-\bar{n}_{i}\left(\frac{\alpha-1}{\alpha}\right)}}{\sum_{j=1}^{N} p_{j}^{\beta}}\right)^{\alpha}=\frac{\sum p_{i}^{\alpha \beta}}{\sum p_{j}^{\beta}}$.

Hence (2.19) becomes

$$
\left(\frac{\sum_{i=1}^{N} p_{i}^{\beta} D^{-n_{i}\left(\frac{\alpha-1}{\alpha}\right)}}{\sum_{j=1}^{N} p_{j}^{\beta}}\right)^{\alpha} \leq \frac{\sum p_{i}^{\alpha \beta}}{\sum p_{j}^{\beta}}<D\left(\frac{\sum p_{i}^{\alpha \beta}}{\sum p_{j}^{\beta}}\right)
$$

which gives (2.17).

\section{Acknowledgement}

We thank the referee for his valuable suggestions.

\section{References}

[1] J. Aczel and Z. Daroczy, Uber Verallgemeinerte quasilineare mittelwerte, die mit Gewichtsfunktionen gebildet sind, Publ. Math. Debrecen, 10 (1963), 171-190.

[2] J. Aczel and Z. Daroczy, On measures of information and their characterizations, Mathematics in Science and Engineering, vol. 115., Academic Press, New York-London, 1975. 
[3] L. L. Campbell, A coding theorem and Renyi's entropy, Information and Control 8 (1965), 423-429.

[4] Z. Daroczy, Generalized information functions, Information and Control 16 (1970), 36-51.

[5] B. Ebanks, P. Sahoo and W. Sander, Characterizations of information measures, World Scientific Publishing Co., Inc., River Edge, NJ, 1998.

[6] A. Feinstein, Foundation of Information Theory, McGraw Hill, New York, 1956.

[7] Gurdial and F. Pessoa, On Useful Information of order $\alpha$, J. Comb. Information and Syst. Sci. 2 (1977), 30-35.

[8] J.F. Havrda and F. Charvat, Qualification method of classification process, the concept of structural $\alpha$-entropy, Kybernetika 3 (1967), 30-35.

[9] D. S. Hooda and U. S. Bhaker, A generalized 'useful' information measure and coding theorems, Soochow J. Math. 23 (1997), 53-62.

[10] F. Jelinek, Buffer overflow in variable lengths coding of fixed rate sources, IEEE 3 (1980), 490-501.

[11] J. N. Kapur, Generalized entropy of order $\alpha$ and type $\beta$, Maths. Seminar, Delhi, 4 (1967), $78-94$.

[12] A. B. Khan, B. A. Bhat and S. Pirzada, Some results on a generalized useful information measure, Journal of Inequalities in Pure and Applied Mathematics 6(4) (2005), Art. 117.

[13] J. C. Kieffer, Variable lengths source coding with a cost depending only on the codeword length, Information and Control 41 (1979), 136-146.

[14] G. Longo, A noiseless coding theorem for sources having utilities, Siam J. Appl. Math. 30 (1976), $739-748$.

[15] B. Mc-Millan, Two inequalities implied by unique decipherability, IEEE Trans. Inform. Theory IT-2 (1956), 115-116.

[16] J. Mitter and Y. D. Mathur, Comparison of entropies of power distribution, ZAMM 52 (1972), 239-240.

[17] Om Parkash and P. K. Sharma, Noiseless coding theorems corresponding to fuzzy entropies, Southeast Asian Bulletin of Mathematics 27 (2004), 1073-1080.

[18] A. Renyi, On Measure of entropy and information, Proc. 4th Berkeley Symp. Maths. Stat. Prob. 1 (1961), 547561.

[19] C.E. Shannon, A Mathematical Theory of Communication, Bell System Tech. J. 27 (1948), 379-423, 623-656.

[20] R. P. Singh, R. Kumar and R. K. Tuteja, Application of Hölder's inequality in information theory, Information Sciences 152 (2003), 145-154.

[21] C. Tsallis, Possible generalization of Boltzmann Gibbs statistics, J. Stat. Phy. 52 (1988), 479.

[22] I. Vajda, Axioms for $\alpha$-entropy of a generalized probability scheme, Kybernetika (Prague) 4 (1968), $105-112$.

Department of Mathematics, Geeta Institute of Management \& Technology, Kanipla-136131, Kurukshetra, Haryana (India).

E-mail: arunchoudhary07@gmail.com 